\title{
PRECISE AND AUTOMATIC 3D ABSOLUTE GEOLOCATION OF TARGETS USING ONLY TWO LONG-APERTURE SAR ACQUISITIONS
}

\author{
Sergi Duque, Alessandro Parizzi, Francesco De Zan and Michael Eineder \\ German Aerospace Center (DLR). Remote Sensing Technology Institute
}

\begin{abstract}
This paper presents a novel approach to determine 3D absolute geolocation of point targets using just two longaperture SAR acquisitions. Moreover, the Zenith Path Delay of both acquisitions is obtained. First results presented here show that the absolute positioning accuracy reaches the submeter level. Index Terms - Absolute geolocation, SAR, Staring Spotlight, TerraSAR-X
\end{abstract}

\section{INTRODUCTION}

Spaceborne SAR systems are already well-known and established systems for Earth monitoring. One of the most popular and classic applications is the generation of Digital Elevation Models (DEMs). The TanDEM-X mission is the most recent mission which aims to obtain a 3D model of the whole Earth with an unprecedented accuracy. The DEM specifications of the TanDEM-X mission correspond to the DTED Level 3, i.e. spacing of 12 meters and height accuracy of 2 meters. This specifications work fine for the major part of the world where the terrain can be considered homogeneous in a spatial resolution of 12 meters. However, urban scenarios are complex to be precisely $3 \mathrm{D}$ modeled by TanDEM-X mission. Alternative SAR based technique like SAR radargrammetry oriented to point targets [1] aim to get a precise and absolute $3 \mathrm{D}$ point cloud. Other SAR coherent techniques like Differential Interferometry [2] and SAR Tomography [3] provide in addition to the point cloud the motion of each point target (PT). General speaking, these techniques select a set of reliable permanent scatterers present on a stack of SAR images and obtain their $3 \mathrm{D}$ geolocation on ground and their motion in case of DinSAR and Tomography. Up to now, obtaining an absolute 3D urban reconstruction using spaceborne SAR imagery presents the following problems:

- Need of a large set of images. This may imply a huge economical and also requires time in case the acquisitions are not in archive.

- Techniques based on SAR interferometry and tomography may need of ground control points.

- For an accurate absolute 3D geolocation, the extra signal path delay due to traveling through mediums dif- ferent than vacuum (i.e.the atmosphere) needs to be taken into account. This implies the necessity of external data to estimate the ionospheric and tropospheric delays seen by the sensor geometry.

- Techniques based on SAR radargrammetry present problems to automatically identify the point targets within the different employed geometries.

The novel method presented in this paper is able to obtain an absolute 3D target geolocation in an automatic way using just two spaceborne Synthetic Aperture Radar (SAR) acquisitions. Moreover, the method is able to derive absolute estimations of the tropospheric delay for both acquisitions. The method described in this paper has been filed for patent application.

The work has been developed using as examples SAR acquisitions from the German satellite TerraSAR-X in Staring Spotlight (ST) mode. This TerraSAR-X acquisition mode consists on steering the antenna to a fixed point on the ground during the whole acquisition time. The azimuth steering angles range from $-2.2^{\circ}$ to $2.2^{\circ}$. The associated azimuth bandwidth is around $38 \mathrm{~K} \mathrm{~Hz}$ yielding an azimuth resolution below $24 \mathrm{~cm}$ [4].

\section{METHOD DESCRIPTION}

The diagram block of proposed method is illustrated in Figure 1. The input to this method consists only in two spaceborne SAR acquisitions with long apertures and different geometries.

The acquisition incidence angle has to be significantly different in order to present sensibility to height changes. It has been demonstrated that using a stereo pair of TerraSAR-X acquisitions in a Spotlight mode with a difference of incidence angles of 26 degrees, it is possible to achieve accuracies over forest areas of few meters [5]. The proposed method is oriented to PTs with high Signal to Clutter Ratio (SCR) and using long-aperture spaceborne acquisitions. Therefore, the expected absolute accuracy for same order of incidence angle difference is in the sub-meter level. According to the diagram flow, first of all, the possible PTs are identified in both images independently. The next step is PT defocus analysis for every 


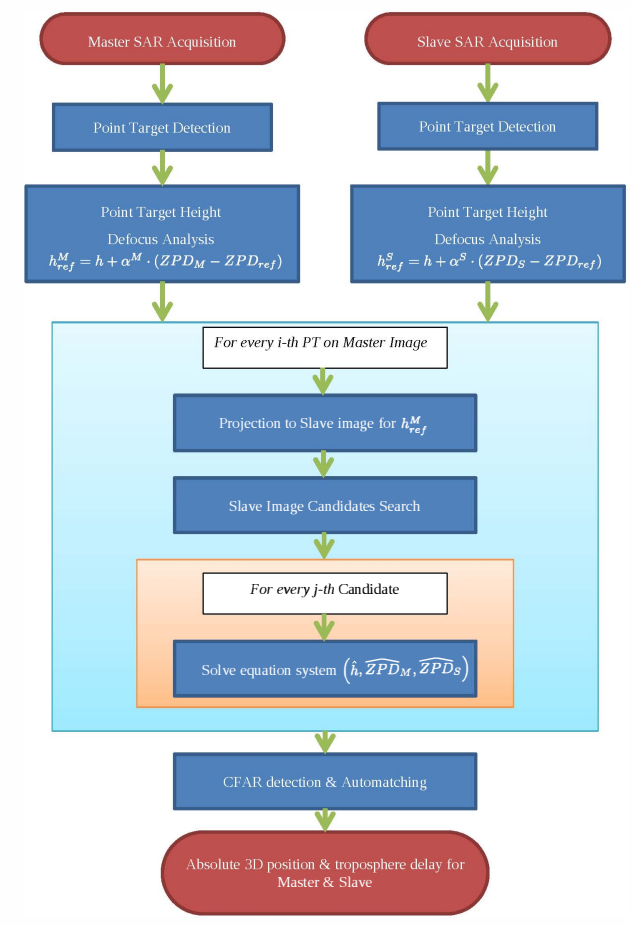

Fig. 1. Diagram flow of the proposed method.

selected PT in each image. According to [6], for a very precise SAR processors, it is possible associate PT azimuth defocus to the height mismatch between real height and height assumed in focusing. This defocussing effect maybe only significant for acquisitions with large curve apertures, such as TerraSAR-X Staring Spotlight acquisitions. The obtained accuracy depends mainly on the azimuth bandwidth (aperture length) and the target SCR.

There is a coupling between height and tropospheric delay as far as focusing parameters are concerned. An unaccounted tropospheric delay during SAR focusing has the same effect as assuming a wrong height. So, from the defocus analysis, it is possible to obtain height estimation for every PT as a function of its tropospheric delay. The linking expression between height and tropospheric delay for a PT in Master image is highly linear. It has the following form

$$
h_{r e f}^{M}=h+\alpha^{M} \cdot\left(Z P D_{M}-Z P D_{\text {ref }}\right),
$$

where $h$ is the height of the target, $h_{r e f}^{M}$ is the derived height from autofocus analysis of Master image assuming a reference tropospheric Zenith Path Delay (ZPD), $Z P D_{M}$ is the true target $\mathrm{ZPD}$ at Master acquisition time, $Z P D_{\text {ref }}$ is the reference ZPD and $\alpha^{M}$ is the linear coefficient for Master image. The expression for the autofocus analysis of selected PTs in the Slave image can be defined likewise with $h_{\text {ref }}^{S}, \alpha^{S}$ and $Z P D_{S}$. From now on, since the tropospheric delay may vary significantly in height, the terms $Z P D_{M}, Z P D_{S}$ and $Z P D_{\text {ref }}$ are referred to a constant reference height, $h_{r e f}$. The translation of ZPD from one height to another can be done using the exponential model given by:

$$
Z P D_{h}=Z P D_{r e f} \cdot e^{\frac{h_{r e f}-h}{H_{0}}}
$$

where $Z P D_{h}$ is the ZPD at a certain height, $Z P D_{\text {ref }}$ is the $\mathrm{ZPD}$ at the reference height and $H_{0}$ is the atmosphere layer thickness, approximately 6 kilometers. The next step is carried out for every PT in the Master image. Every target is projected in the Slave geometry taking into account its rangeazimuth Master position and the derived height at $Z P D_{\text {ref }}$. Then, the search for point target candidates in the Slave image referring to the same target takes place. Ideally, if there was no influence of the unaccounted tropospheric delay, the corresponding point target should appear on the Slave image along a line. Notice that this line is not parallel to range direction due to the heading angle difference between acquisitions. Its direction is a combination of range and azimuth. The possible values of tropospheric ZPD can be reasonably bounded. Taking into account this possible variation of ZPD and the autofocus height error derived in [6] is possible to determine an area where the point target should appear in the Slave image. The PTs from the Slave image falling into this area are the candidates. Figure 2 illustrate the process of mapping a PT from the Master image to the Slave image and looking for its matching candidates. Then, the next step is to solve a lin-

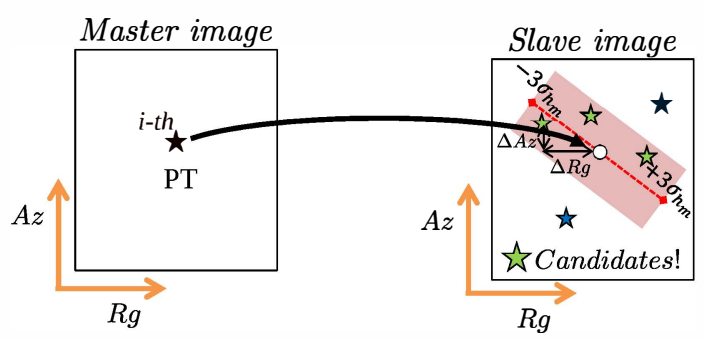

Fig. 2. Schema of candidate search in the Slave image for a point target in the Master image.

ear equation system for each candidate. The equation system is composed by four equations. Two of them are regarding the Azimuth and Range displacement $(\Delta A z, \Delta R g)$ and the other two refer to the link between point target height and the tropospheric delay in Master and Slave acquisitions. Thus, the 
equation system can be express by

$$
\begin{aligned}
\Delta R g= & K_{R g} \cdot\left(h-h_{r e f}^{M}\right)+ \\
& \beta_{R g}^{M} \cdot\left(Z P D_{M}-Z P D_{r e f}\right)+ \\
& \beta_{R g}^{S} \cdot\left(Z P D_{S}-Z P D_{r e f}\right) \\
\Delta A z= & K_{A z} \cdot\left(h-h_{r e f}^{M}\right)+ \\
& \beta_{A z}^{M} \cdot\left(Z P D_{M}-Z P D_{r e f}\right) \\
h_{r e f}^{M}= & h+\alpha^{M} \cdot\left(Z P D_{M}-Z P D_{r e f}\right) \\
h_{r e f}^{S}= & h+\alpha^{S} \cdot\left(Z P D_{S}-Z P D_{r e f}\right) .
\end{aligned}
$$

Here, $K_{R g}, K_{A z}, \beta_{R g}^{M I}, \beta_{R g}^{S}, \beta_{A z}^{M}, \alpha^{M}$ and $\alpha^{S}$ can be considered constant parameters and can be numerically computed. The equation system is composed of four equations and has three unknowns, the targets height, $h$, and its tropospheric delays for Master and Slave acquisitions, i.e. $Z P D_{M}$ and $Z P D_{S}$ respectively. Since the equations are not linearly dependent, it is possible to find a solution and retrieve the unknowns. The problem at this point is that they may be multiple candidates for each $i$-th Master PT under test. Figure 3 shows two SAR acquisitions of TerraSAR-X in Staring Spotlight mode over a building facade with two different incidence angles, Figure 3(a) refers to the Master image with an incidence angle of $36^{\circ}$ while Figure 3(b) is the Slave image acquired with $54^{\circ}$. As an example, a selected point is marked

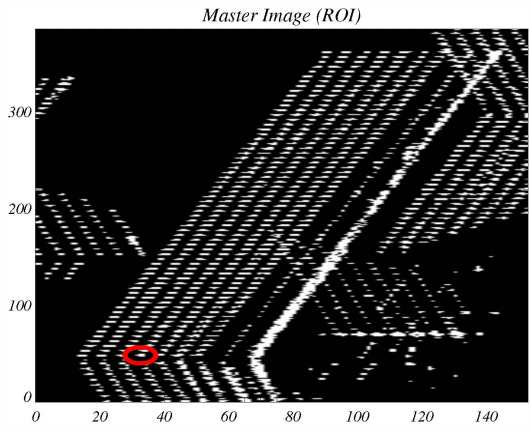

(a)

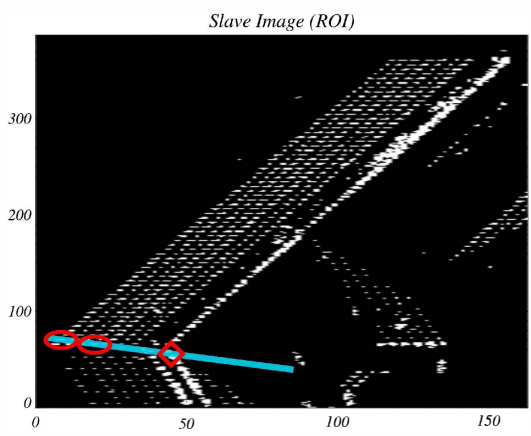

(b)

Fig. 3. Master and Slave facades.

by a red ellipse in the Master image (Figure 3(a)) and its pro- jection in Slave geometry is marked by a red diamond in Figure 3(b). The search area taking into account the derived autofocus height, its accuracy and reasonable possible values of tropospheric delays for Master and Slave is marked by a cyan trapezoid in the Slave image. Two candidates appear on the Slave image marked by red ellipses. According to the flow chart, the next step is to solve the equation system for all the possible candidates for each selected PT in the Master image. Afterwards, it is possible to exploit the spatial behavior of tropospheric delay to determine which candidates are the true point targets from the Master image. The tropospheric delay varies slowly in the horizontal plane. This effect is exploited in this method to achieve the automatching. The tropospheric delay horizontal variation can be considered negligible for distances of few hundred meters.

All the found solutions of ZPD for Master and Slave are analyzed together for all candidates within a relatively small spatial window. The candidates who give a ZPD solution for Master and Slave around the main distribution will be those ones that really match the point targets in the Master image.

\section{FIRST RESULTS}

Here, the authors present a first result retrieving the heights of the point targets located over the building facade shown previously in Figure 3. The atmospheric delay is also obtained for the area at the set reference height of $100 \mathrm{~m}$. Here the authors have taken into account the Solid Earth Tides and the ionospheric delays. In case they are not taken into account, they will produce a small bias of few centimeters on the height estimation. The master and slave incidence angles are $36^{\circ}$ and $54^{\circ}$ respectively, yielding a difference of $18^{\circ}$. The heading angle variation between Master and Slave acquisition is $3.5^{\circ}$. A range and azimuth displacement of 1 meter w.r.t. expected position in Slave image correspond to a height variation of 1.9 meters and -12.37 meters respectively. The range and azimuth resolutions of the TerraSAR-X Staring Spotlight acquisition mode are $59 \mathrm{~cm}$ and $23 \mathrm{~cm}$ respectively. Figure 4 shows the 2D histogram of ZPD at the reference height for Master and Slave acquisitions. In this case three Gaussian distributions appear due to the regular pattern of the point targets in the facade. However, there is one distribution with much more energy. Here, we have selected a specific facade which follows some point target pattern distribution. Normally, in a more heterogeneous test, the expected 2D histogram will present a Gaussian distribution surrounded by noise. The noise power will be dependent on the point target density of the scene. The red circle at Figure 4 represents the ZPD at $h_{r e f}$ estimated using weather models [7]. Table 1 shows the good agreement between the ZPD estimated using the weather model and the described method.

Figure 5 shows the obtained heights using the proposed method (Figure 5(a)) and a comparison with a LiDAR measurement (Figure 5(b)). The probability of detection is close 


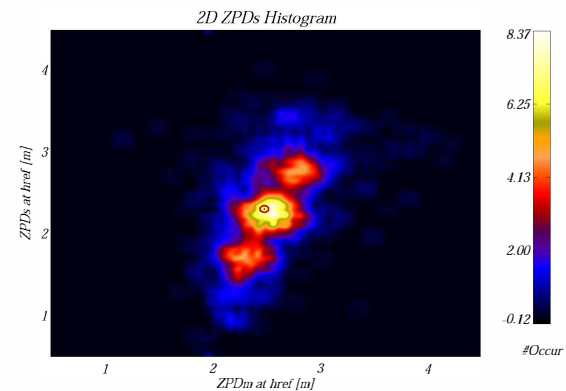

Fig. 4. 2D histogram of ZPD at $h_{r e f}$ for Master and Slave acquisitions.

\begin{tabular}{|l||c|c|c|}
\hline & Weather Model & Our Method & Diff. \\
\hline Master & $2.49 \mathrm{~m}$ & $2.55 \mathrm{~m}$ & $-6.4 \mathrm{~cm}$ \\
\hline Slave & $2.31 \mathrm{~m}$ & $2.28 \mathrm{~m}$ & $2.2 \mathrm{~cm}$ \\
\hline
\end{tabular}

Table 1. ZPD at height reference estimated by a weather model and the described method.

to $90 \%$, yielding a $10 \%$ of false alarm rate. The false alarms can be seen as outliers in Figure 5(b) and Figure 5(a). The height bias and standard deviation w.r.t. LiDAR measurement are calculated using the good detections, yielding a bias of $-18.4 \mathrm{~cm}$ and a standard deviation of $15.7 \mathrm{~cm}$.

\section{SUMMARY AND CONCLUSIONS}

This paper has presented a novel method for absolute 3D geolocation using just two long-aperture SAR acquisitions. In addition, the tropospheric delays can be estimated for both acquisitions as a by product. The method has been tested over a building facade with accuracies in the sub-meter level. The proposed method can be used to generate a urban point cloud with just two acquisitions, reducing costs and acquisition time. In order to extend the method to a complete scene, the processing needs to be carried out block size due to the assumption of low-pass spatial behavior of tropospheric delay.

\section{REFERENCES}

[1] K. Goel and N. Adam, "Three-dimensional positioning of point scatterers based on radargrammetry," Geoscience and Remote Sensing, IEEE Transactions on, vol. 50, no. 6, pp. 2355-2363, June 2012.

[2] Stefan Gernhardt and Richard Bamler, "Deformation monitoring of single buildings using meter-resolution sar data in psi," ISPRS Journal of Photogrammetry and Remote Sensing, vol. 73, pp. 68-79, 2012.

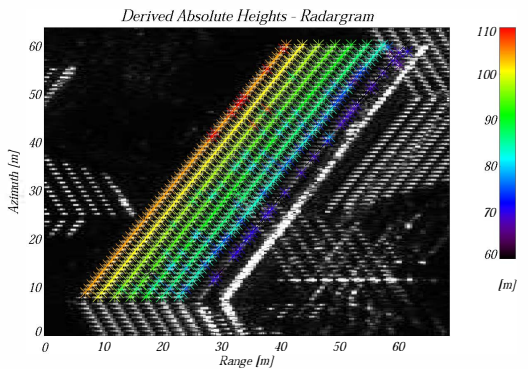

(a)

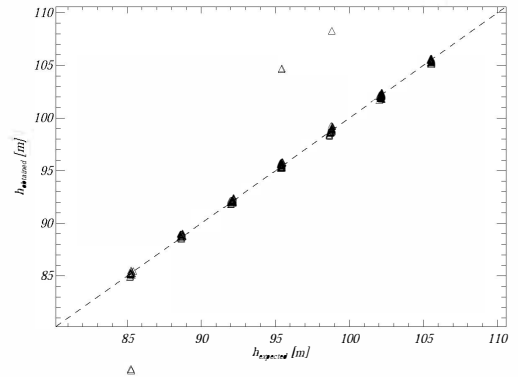

(b)

Fig. 5. Obtained using the proposed method (a) and LiDAR comparison (b).

[3] Xiao Xiang Zhu and Richard Bamler, "Superresolving sar tomography for multidimensional imaging of urban areas: compressive sensing-based tomosar inversion," Signal Processing Magazine, IEEE, vol. 31, no. 4, pp. 51-58, 2014

[4] P. Prats-Iraola et al, "On the Processing of Very High Resolution Spaceborne SAR Data," Geoscience and Remote Sensing, IEEE Transactions on, vol. 52, no. 10, pp. 6003-6016, Oct 2014

[5] K. Gutjahr, R. Perko, H. Raggam, and M. Schardt, “The Epipolarity Constraint in Stereo-Radargrammetric DEM Generation," IEEE Transactions on Geoscience and Remote Sensing, vol. 52, no. 8, pp. 5014-5022, Aug 2014.

[6] S. Duque, H. Breit, U. Balss, and A. Parizzi, “Absolute Height Estimation Using a Single TerraSAR-X Staring Spotlight Acquisition," Geoscience and Remote Sensing Letters, IEEE, vol. 12, no. 8, pp. 1735-1739, Aug 2015.

[7] Xiaoying Cong, U. Balss, M. Eineder, and T. Fritz, "Imaging Geodesy - Centimeter-Level Ranging Accuracy With TerraSAR-X: An Update," Geoscience and Remote Sensing Letters, IEEE, vol. 9, no. 5, pp. 948-952, Sept 2012. 